\title{
A case of paradoxical brain embolism presenting as a typical lacunar stroke
}

\author{
Gian Paolo Anzola $^{1^{*}}$, F. Casilli $^{2}$, E. Onorato $^{2}$ \\ ${ }^{1}$ Service of Neurology, S.Orsola Hospital FBF, Brescia, Italy; *Corresponding Author: gpanzola@numerica.it \\ ${ }^{2}$ Department of Cardiology, S.Orsola Hospital FBF, Brescia, Italy.
}

Received 18 November 2009; revised 8 January 2010; accepted 15 January 2010.

\begin{abstract}
A 59 year old white male presented with a clinical picture typical of lacunar stroke. However, a thorough diagnostic work up showed that the most probable mechanism of stroke was paradoxical brain embolism through a patent foramen ovale. The clinical-neuroradiological mismatch that ultimately lead to the correct interpretation is discussed.
\end{abstract}

Keywords: Lacunar Stroke; Patent Foramen Ovale

\section{INTRODUCTION}

Lacunar strokes are usually recognizable on clinical grounds when one of the "typical" clinical presentations identified by Mille-Fisher [1] is coupled with the lack of obvious cardiac embolic sources or major atherosclerotic lesions of the supplying vessels. Conventional neuroimaging (CT scan or T2 weighted MRI) is considered useful to exclude non vascular lesions, but with little additive value in identifying the cause of stroke.

We report a case of an apparently typical lacunar stroke in whom, on the contrary, Diffusion Weighted MRI was of paramount importance in suggesting the correct cause.

\section{CASE REPORT}

A 59 year old male caucasian craftsman was admitted with a two day history of right sided weakness of sudden onset, difficulty with walking, but no change in consciousness, headache, subjective visual complaints, or cutaneous sensory symptoms.

He had a history of long standing non insulin dependant diabetes and hypertension. Two years earlier he had sustained transient numbness and loss of strength affecting his left arm and lasting for some days.

On admission he appeared fully alert and aware. He had no aphasia, visual field defects, or dysphagia, and a barely appreciable slurring of speech with right sided mild hemiparesis (arm drift and slow leg fall) sparing his face. There was no sensory disturbance.

Arterial blood pressure was 160/90, EKG showed sinus rhythm.

Routine blood tests revealed total cholesterol 220 $\mathrm{mg} / \mathrm{dl}$, blood sugar $143 \mathrm{mg} / \mathrm{dl}$, creatinin $0,8 \mathrm{mg} / \mathrm{dl}$, DDimer $>600 \mathrm{ng} / \mathrm{ml}($ normal $<275 \mathrm{ng} / \mathrm{ml}$ ).

Echo Doppler disclosed only minimal narrowing of carotid walls, transcranial Doppler $>20 \%$ asymmetry with reduced mean flow velocity in the left middle cerebral artery. Transthoracic echocardiography showed mild left ventricular hypertrophy.

The clinical picture of pure motor brachio-crural hemiparesis ( with no "cortical" features) in conjunction with diabetes and hypertension in the absence of significant carotid disease and no obvious potential cardiac sources of embolism led to the provisional diagnosis of lacunar stroke. A neuroimaging procedure was then performed.

T2 weighted FLAIR MRI showed multiple spot like lesions affecting the basal ganglia, the internal capsule and the white matter of the corona radiata bilaterally, which seemed to confirm the diagnosis of small vessel disease. However, DWI sequences, besides showing a bright spot in the posterior limb of the left internal capsule, which was probably responsible for the clinical pictures also disclosed a number of further recent lesions scattered in the subcortical and deep white matter of both hemispheres, compatible with multiple microembolic occlusions of perforating vessels.

Following MRI, it was decided to proceed to intra-arterial arteriography, which ruled out significant lesions of the aortic arch and the intracranial cerebral arteries.

A Doppler study of lower limbs revealed very minor irregularities of the calf veins with no evidence of overt thrombosis. A transcranial bubble test showed a rightto-left shunt with 30 bubbles in basal conditions and $>100$ with curtain pattern following Valsalva strain. 


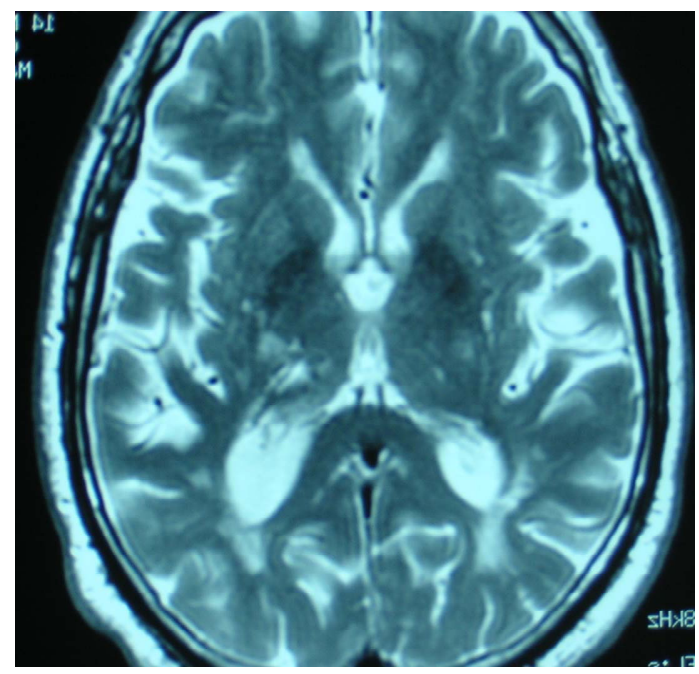

(a)

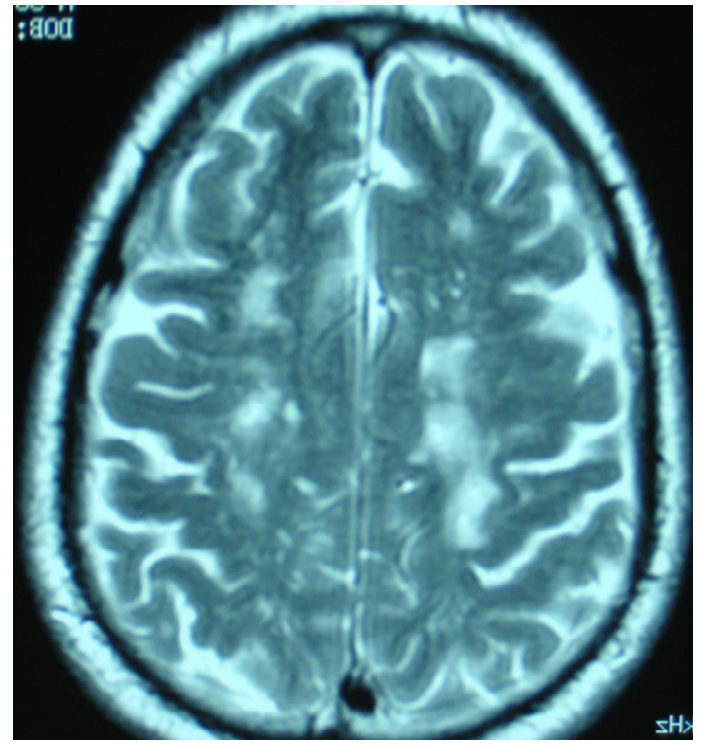

(b)

Figure 1. T2 FLAIR MRI showing multiple spotlike lesions in the basal ganglia, internal capsule and thalamus (panel A). In the hemispheric white matter the abnormalities tend to become confluent (panel B).

This finding, together with the elevation in plasma D-dimer, the results of venous Doppler and the appearance of DWI MRI, were considered compatible with brain paradoxical embolism through a patent foramen ovale. During the entire hospital stay continuous ECG telemetry revealed no significant abnormality.

Because of the diffuse white matter abnormalities, and the history of diabetes and hypertension, long term oral anticoagulation was deemed unsafe. Therefore, one month later following a short course of low molecular weight heparin, the patient underwent a successful transcatheter closure of the patent foramen ovale with a Cardia In- trasept device. He has since fared well and at one year follow up he has resumed his job with only mild clumsiness in the finger movements of his right hand.

\section{DISCUSSION}

This case had a presentation typical of a lacunar syndrome, entailing a pure motor stroke in a hypertensive, diabetic patient without obvious arterial or cardiac embolic sources. The lacunar hypothesis, as put forward by Miller-Fisher, assumes that specific clinical syndromes are produced by infarctions in the territory of a single perforating artery at the base of the brain or in the brainstem as a consequence of local phenomena such as lipohyalinosis or microatheroma [1].

With the advent of CT and MRI neuroimaging since the beginning of the '90s [1,2], the first part of the hypothesis was shown to be substantially correct whereas it proved much more difficult to demonstrate the second part. About $10 \%$ of lacunar syndromes are due to non ischemic brain damage [3], but even when the underlying pathological cause is ischemic it has proved difficult to identify a single mechanism. The traditional common teaching of local occlusion promoted by diabetes and hypertension is not supported by epidemiological evidence, as hypertension and diabetes are no more common in lacunar than in non lacunar strokes [4-6]. On the other hand, Miller Fisher himself admitted and postulated that some of his cases might have been due to embolism, mainly on the basis of the patency of the arteries supplying the infarcted tissue [1]. Therefore, even in recent textbooks [7] the precise etiological interpretation remains doubtful, although it is often suggested that cardiogenic embolism is a very rare cause of lacunar syndromes $[2,4,5]$. Contrary to this assumption Wessel et al., by systematically investigating patients with DWI- MRI, found that 14 (19\%) out of 73 patients with lacunar syndromes had multiple recent lesions in different areas, thus suggesting cardiac embolism as the likely cause [8], although they only reported atrial fibrillation as potential embolic source in 3 patients. More recently Ueno et al. [9] included RLS as a potential cardiac source of emboli and found that compared with the $32 \%$ prevalence found in healthy controls, lacunar patients had a significantly higher 41\% prevalence when hypertension and/or diabetes were present and a still higher $82 \%$ prevalence when neither risk factor was present. However they did not correlate the presence of RLS with the MRI findings [9].

In the present case, we were able to demonstrate both the embolic nature of the stroke and the underlying mechanism. Despite the presence of both hypertension and diabetes the finding of a massive shunt associated with the elevation of D-dimer, the abnormalities of venousechography and the lack of arrhythmia are highly sug- 


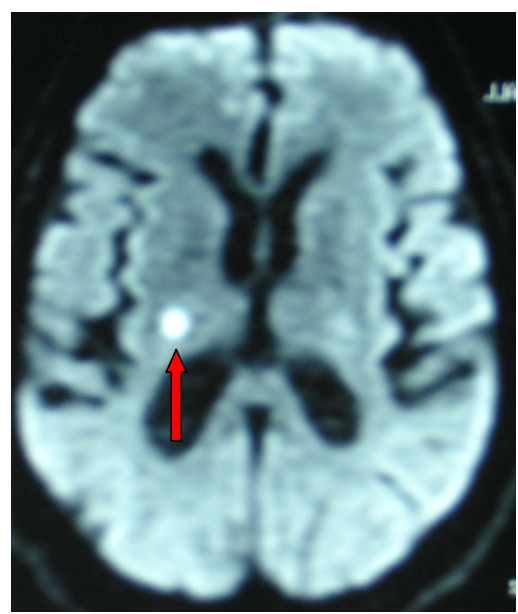

(a)

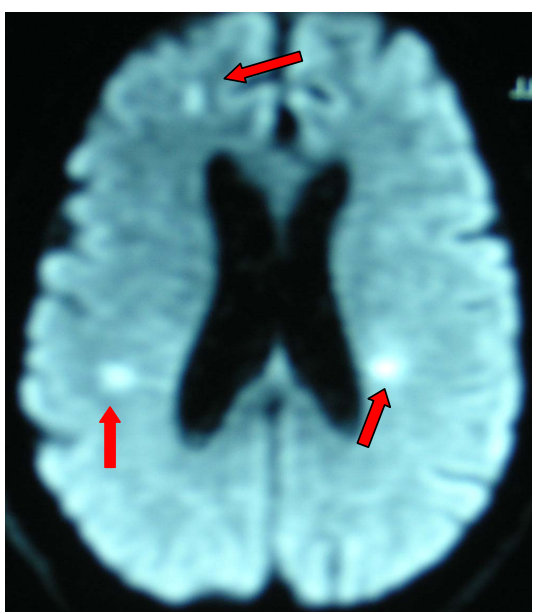

(b)

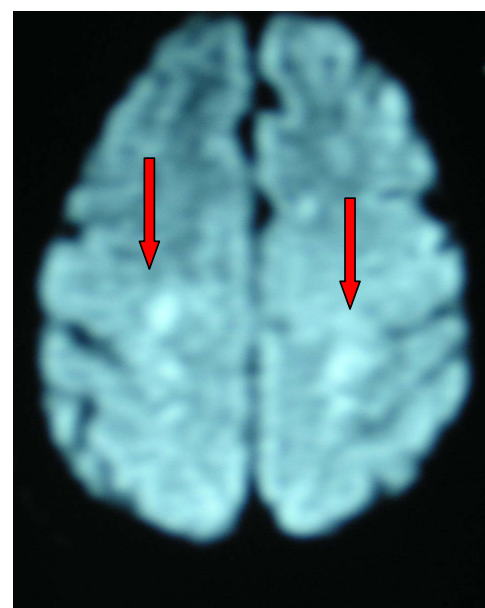

(c)

Figure 2. DWI MRI showing multiple recent bright lesions in the left posterior internal capsule (arrow, panel (a)) and bilateral hemispheric white matter (arrows, panels (b) and (c)).

gestive of paradoxical brain embolism (PBE) as the most probable explanation of the embolic pattern seen on MRI.

PBE is considered a likely cause of stroke in young patients when more common causes are excluded. However, it can be important also in older patients [10] and as an independent factor even when other cerebrovascular risk factors are present [11].

Therefore, since patent foramen ovale can be found in as many as $25 \%$ of stroke patients with essentially no specific clinical profile [12], PBE should be considered within the differential diagnosis even when apparently more obvious alternative diagnoses are contemplated, once DW-MRI has suggested the presence of an embolic ischemic pattern.

In conclusion, this case highlights on one hand the low predictive value of the clinically defined lacunar picture in identifying the cause of the stroke and on the other hand the usefulness of the systematic application of the most recent techniques of neuroimaging. In fact it was the MRI appearance that pointed towards a systematic search for embolic sources that would probably have otherwise been overlooked.

\section{Acknowledgement}

The Authors wish to thank Dr Edward Stonehill for his friendly cooperation in improving the text and revising the English.

\section{REFERENCES}

[1] Bamford JM and Warlow CP. (1988) Evolution and testing of the lacunar hypothesis. Stroke, 19, 1074-1082.

[2] Boiten J and Lodder J. (1991) Lacunar infarcts. Pathogenesis and validity of the clinical syndromes. Stroke, 22,
1374-1378.

[3] Anzalone N and Landi G. (1989) Non ischaemic causes of lacunar syndromes: prevalence and clinical findings. $J$ Neurol Neurosurg Psychiatry, 52, 1188-1190. doi:10.1136/jnnp.52.10.1188

[4] Lodder J,Bamford JM, Sandercock PAG, Jones LN and Warlow CP. (1990) Are hypertension or cardiac embolism likely causes of lacunar infarction? Stroke, 21, 375-381.

[5] Sacco SE, Whisnant JP, Broderick JP, Philips SJ and O’Fallon WM. (1991) Epidemiological characteristics of lacunar infarcts in a population. Stroke, 22, 1236-1241.

[6] Jackson C and Sudlow C. (2005) Are lacunar strokes really different? A systematic review of differences in. risk factor profiles between lacunar and nonlacunar infarcts. Stroke, 36, 891-901. doi:10.1161/01.STR.0000157949.34986.30

[7] Warlow CP, Dennis MS, van Gijn J, Hankey GJ, Sandercocl PAG, Bamford JM and Wardlaw JM (2003) Stroke: a practical guide to management. 2nd Edition, Blackwell Publishing, 133-138.

[8] Wessels T, Röttger C, Jauss M, Kaps M, Traupe H and Stolz Erwin. (2005) Identification of embolic stroke patterns by diffusion-weighted MRI in clinically defined lacunar stroke syndromes. Stroke, 36, 757-761. doi:10.1161/01.STR.0000158908.48022.d7

[9] Ueno Y, Kimura K, Iguchi Y, Shibazaki K, Inoue T and Urabe T. (2007) Right-to-left shunt and lacunar stroke in patients without hypertension and diabetes. Neurology, 68, 528-531. doi:10.1212/01.wnl.0000253197.83777.c7

[10] Handke M, Harloff A, Olschewski M, Hetzel A and Geibel A. (2007) Patent foramen ovale and cryptogenic stroke in older patients. The New England Journal of Medicine, 357, 2262-2268. doi:10.1056/NEJMoa071422

[11] Homma S, DiTullio MR, Sacco RL, Sciacca RR and Mohr JP. (2004) Age as a determinant of adverse events in medically treated cryptogenic stroke patients with patent foramen ovale. Stroke, 35, 2145-2149. doi:10.1161/01.STR.0000135773.24116.18 
[12] Jauss M, Wessels T, Trittmacher S, Allendörfer $\mathrm{J}$ and Kaps M. (2006) Embolic lesion pattern in stroke patients with patent foramen ovale compared with patients lack- ing an embolic source. Stroke, 37, 2159-2161. doi:10.1161/01.STR.0000231645.22128.ab 